\title{
High-Fidelity Simulation of Large-Scale Structures
}

\author{
Christoph Hoffmann, Ahmed Sameh, and Ananth Grama \\ Department of Computer Sciences, Purdue University, W. Lafayette, IN 47907
}

\begin{abstract}
Active structures capable of responding to external stimulii represent the emerging frontier in structural design. Robust and real-time sensing, control, and actuation pose fundamental challenges that must be addressed in this context. As part of an ambitious project funded by the National Science Foundation, researchers at Purdue, Rice, Florida State, and the Catholic (Belgium) Universities have undertaken development of these core technologies. Over the past 18 months, considerable progress has been made in the areas of model reduction and control, sensing, and simulation-based validation. This paper describes our results in highfidelity simulations of large structures, subject to various (mechanical and thermal) stresses.

A high-fidelity simulation infrastructure poses a number of challenges. These include geometric modeling (generating a suitable mesh for the structure), physical modeling (developing mathematical models for coupling between various phenomena, specifying material properties), computational modeling (developing efficient numerical schemes and their parallel formulations), and appropriate visualization techniques. We have made fundamental contributions in each of these areas. Here, we overview some of our major contributions, along with sample simulations of existing structures. As part of our ongoing work, we also aim to perform a high-fidelity simulation of the tragic World Trade Center (WTC) crash. To this end, we have developed, from available blueprints, a highly resolved geometric model of the WTC. We also aim to complement all of our computational studies with detailed experimental validation on fullscale structures at the Bowen Lab for Structural Engineering. To the best of our knowledge, this is the first comprehensive effort to fully integrate simulation and modeling with sensing, control, and actuation in an experimental setting. In this sense, we believe that this project is a novel realization of the concept of dynamic data-driven application systems in the realm of large-scale structures.
\end{abstract}

\section{Introduction}

Physical processes are described primarily using mathematical models that are used to simulate the behavior of the underlying processes. Often, they are also used to modify or control system behavior. In this framework, there is an ever increasing need for improved accuracy, which leads to models of higher complexity. The basic motivation for system approximation is the need, in many instances, 
for a simplified model of a dynamical system, which captures the main features of the original complex model. This need arises from limited computational, accuracy, and storage capabilities. The simplified model may then be used in place of the original complex model, either for simulation, or control. As sensor networks and embedded processors proliferate our environment, technologies for such approximations and real-time control emerge as the next major technical challenge.

As part of an ambitious NSF-funded project, we are exploring efficient and robust methods for producing reduced order models of large state-space systems, developing algorithms for real-time control, mechanisms for actuation, and systems infrastructure for affecting sensing, control, and actuation. To this end, we have made several fundamental contributions in the area of control $[6,5,4,3]$, sensing $[1,2]$, and modeling [7,8].

A critical aspect of our study is the validation of underlying model reduction and control mechanisms using detailed computational simulations as well as experimental studies. The objectives of this are many-fold - we intend to precisely quantify errors in model reduction by simulating a hierarchy of progressively coarser models. To this end, we have developed a series of benchmarks, derived from a well-known structure (sanitized and shown in Figure 1). These benchmarks, derived from blueprints of the structure, have been made available over the public domain with the goal of comparing and validating results of control algorithms and model reduction. A second objective of developing a comprehesive simulation framework is to provide mechanisms for incorporating control algorithms and variable damping to study structural response. Finally, we have used our framework as a sophisticated diagnostic/prognostic tool for structural failure. Our work on analysis of the Pentagon crash (Figure 2) is widely accepted as a benchmark study in this regard.

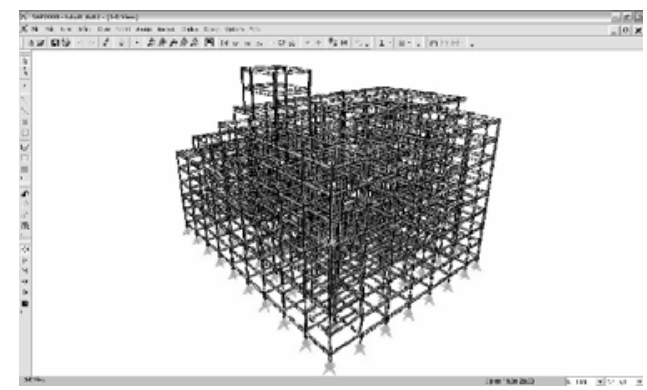

Fig. 1. Illustration of one of the benchmark structures used for model reduction and control

In this paper, we describe major technical challenges in high-fidelity simulations, our approach to addressing these challenges, and our recent results. We also describe ongoing work and the use of experimental studies to fully validate 


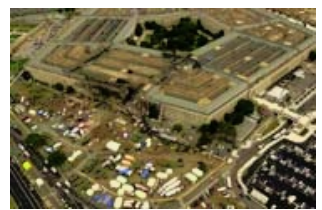

(a) Aerial view of damage to the Pentagon building

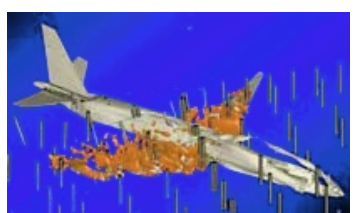

(b) Simulated aircraft impact on $\mathrm{RC}$ columns in the building

Fig. 2. High fidelity simulation of the Pentagon crash performed in our group has yielded significant insights into structural properties of reinforced concrete and design of resilient buildings

our computational results. To the best of our knowledge, this is the first comprehensive effort to fully integrate simulation and modeling with sensing, control, and actuation in an experimental setting. In this sense, we believe that this is a novel realization of the concept of dynamic data-driven application systems in the realm of large-scale structures.

\section{Technical Challenges}

A number of technical challenges must be overcome to develop a comprehensive simulation framework. We have addressed these challenges and continue to explore more efficient techniques. We outline here some of our results in these areas.

\subsection{Geometric Modeling - Meshing}

Meshing represents one of the most time-consuming (labor intensive) part of the simulation process. The fidelity of a simulation is critically influenced by the mesh size and quality. A coarse discretization used for the elements of a Lagrangian mesh reduces the fidelity for contact and yielding behavior. Similarly, a coarse discretization used for the elements of an Eulerian mesh reduces the fidelity for mass transfer computations for the advection process, EulerLagrangian coupling, and the accuracy of fluid flow. In our simulation framework, we have created our own set of meshing tools and a detailed meshing methodology. This allows us to balance the desire for high resolution meshes, and the accuracy so obtained, with the necessity to accommodate the limitations of the underlying computing platform.

A completely automated meshing program that derives an FEA mesh from a geometric model is a significant undertaking. However, much can be accomplished when the meshing is semi-automatic. Since we typically combine separately meshed parts, we generate the mesh in two passes: the first pass generates a mesh description in an intermediate format that is independent of the underlying simulation engine. A second pass then translates the intermediate representation into a form that is suitable for input to selected simulation engines (in 
our case, LS_Dyna). Thus, we can easily change the FEA package, for instance when comparing fidelity and performance of different FEA systems. We have extensive experience with this methodology and have successfully used it to generate meshes for our test structures, benchmark structures, as well as existing buildings (the Pentagon, Bank One building, and the World Trade Center).

\subsection{Physical Modeling - Coupled Simulations}

In addition to traditional issues of developing appropriate models for material behavior and determining suitable material properties, one of the major challenges arises from a coupling of multiple external stimulii. This happens, for instance, when structural failure is induced by thermal stresses. In such cases, it is necessary to simulate all of the physical processes in a tightly integrated manner. We show results from one such simulation performed by us in Figure 3. In this simulation of a fire on the 25th floor of the Bank One building in downtown Indianapolis, complete structural failure is caused by failure of a few critical beams. The underlying challenge is that the temporal and spatial discretizations associated with various phenomena potentially differ by several orders of magnitude. In the case illustrated here, the fire code used had a much finer spatial discretization and coarser temporal discretization than the structural counterpart. However, changes in structure critically impact progression of the fire namely that changes in structure are critical for providing air for feeding the fire. A naive coupling of the two codes requires us to solve the Navier-Stokes equation for compressible fluid flow (air), the combustion model along with the
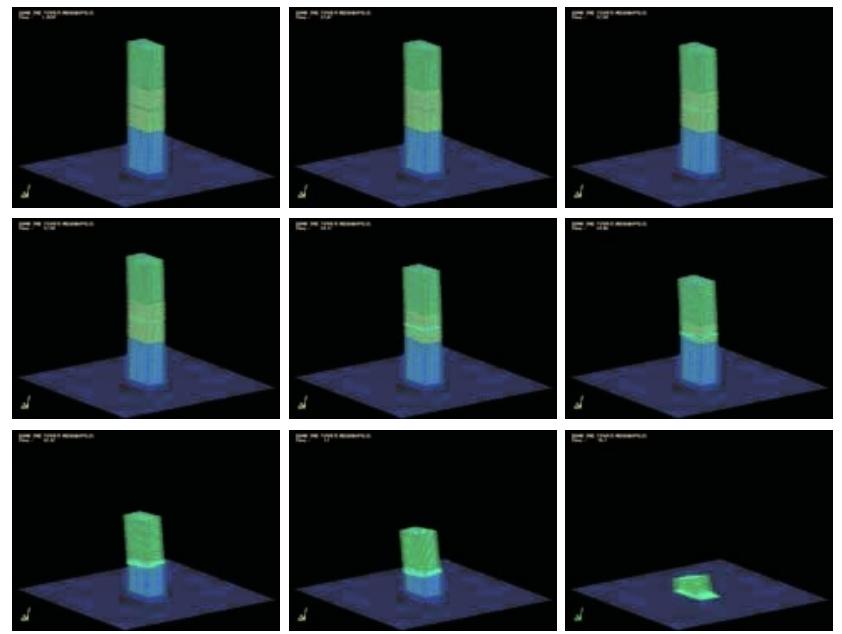

Fig. 3. Simulation of heat-induced structural failure - a fire on the 25th floor of the Bank-One tower in downtown Indianapolis is simulated using a coupled firestructure code (wireframe model shown here). The entire simulation is available at http://www.cs.purdue.edu/homes/ayg/SIM 
flow of the fuel, the structural model, and the solid body motion (for failed components), each at the finest granularity. This renders the simulation extremely computationally expensive.

The approach outlined about corresponds to an explicit timestepping scheme (which was also used in our simulation). While this scheme is generally easier to implement when using multiple production codes, stability in such schemes mandates the use of small timesteps. In contrast, implicit schemes permit use of larger timesteps, however, they require solution to a more complex system of equations. Solutions to such systems pose formidable challenges from a numerical standpoint. We are currently exploring a number of these research issues in the context of our simulation of the WTC crash (Section 3).

\subsection{Computational Modeling - Numerical Methods and Parallelism}

High-fidelity models, of the kind used in our simulations generate extremely large systems, which are typically solved iteratively. This puts the emphasis of timeto-solution on efficient numerical methods (preconditioners for iterative solvers) and their parallel formulations.

Our work on preconditioning linear systems arising from our structural simulations has resulted in extremely effective parallizable preconditioners. These preconditioners, based on our Spike [9] algorithm, are capable of fast convergence, low FLOP counts, and high concurrency. We have characterized the performance of solvers based on our preconditioner for a variety of meshes. We have studied the impact of various elements (eg., shell, beam, column) on the conditioning of the matrix and overall solution time. We demonstrate the superior performance of our methods in comparison to various existing solvers.

The size and complexity of systems resulting from our models necessitate the efficient use of powerful parallel platforms. For example, a single simulation instance of our Pentagon Crash, with one million nodes (3 d.o.f. per finite element node) over 0.25 seconds of real time takes over 68 hours on a dedicated 8 processor IBM Regatta SMP. A coarser model with $300 \mathrm{~K}$ nodes over $0.2 \mathrm{~s}$ of real time takes approximately 20 hours. In addition to the IBM SMP, we have also ported our codes to Linux/Itanium clusters. We have demonstrated excellent parallel efficiency for our codes for a variety of simulations. The computational requirements of these simulations provide strong motivation for effective error-bounded model reduction.

\subsection{Visualization - The Human Interface}

Large scale simulations produce massive multidimensional datasets. Although some scalars can be examined and presented using tables and graphs, 3D visualizations have long been used as powerful means of conveying such results. In the case of FEA simulations that analyze the mechanical interaction of entities under the extreme conditions of a high-kinetic-energy impact, visualization is an indispensable tool. In order to capitalize on high-fidelity finite element modeling, material and contact behavior, the visualization must have high fidelity as well. 


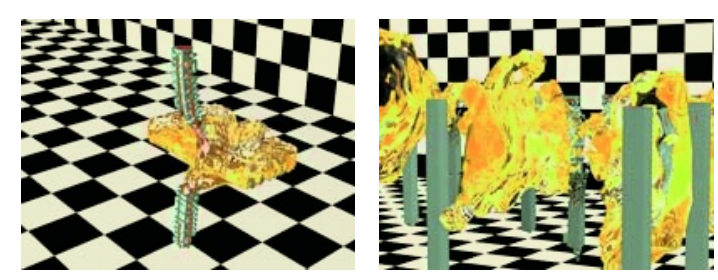

Fig. 4. Visualization of liquid, including reflection and refraction, for fuel flow in an aircraft crash

To affect a realistic and efficient visualization system, we build upon existing commercial solutions using plugins for data transfer, selection, material properties, and a variety of novel visualization techniques. We illustrate one such technique in Figure 4, which shows the flow of fuel through a set of columns. Such visualizations, developed in collaboration with experts in structural engineering, are very useful in understanding structural failure. More details of these techniques is provided in $[7,8]$.

\section{Discussion and Ongoing Work}

An immediate goal of our study is to perform high-fidelity simulations of the tragic World Trade Center (WTC) collapse, with a view to understanding, precisely, the structural cause of failure. We have obtained detailed blueprints of the structure and generated highly resolved geometric models (Figure 5). A major difficulty with this simulation is the coupling of thermal effects with mechanical stresses. This arises from the multitude of phenonema (fuel transport, fuel combustion including air-flow, combustibles in the building, mechanical impact, structural failure, etc.), timescales (all of these phenomena span over three orders of magnitude in time), and ambient factors (air-flow, temperature). Drawing on our initial simulation of the Bank-One building, and our experience in such simulations, we expect to demonstrate these simulations in the near-term.

A unique aspect of our study is that we aim to validate all of our computational results with detailed experimental studies. This effort relies heavily on extensive facilities available at the Bowen Lab for Structural Engineering at Purdue University. Leveraging other grants, we have built a full-scale threestorey test structure $(30 \times 50 \times 35$ feet $)$, shown in Figure 6 . A number of tests are planned on this structure, leading to the eventual testing to failure. These tests include low-frequency high-amplitude lateral and vertical loading (using hydraulic rams), low-amplitude tests for validating modes, and finally, impact of active damping on structural characteristics.

Our immediate goal in this area is to develop a real-time sensing network for measuring displacements, acceleration, and strain, at various points in the structure. For sensing displacements, we use laser sensors from Acuity Research (http://www.acuityresearch.com/contact-us.shtml). These sensors have a range 

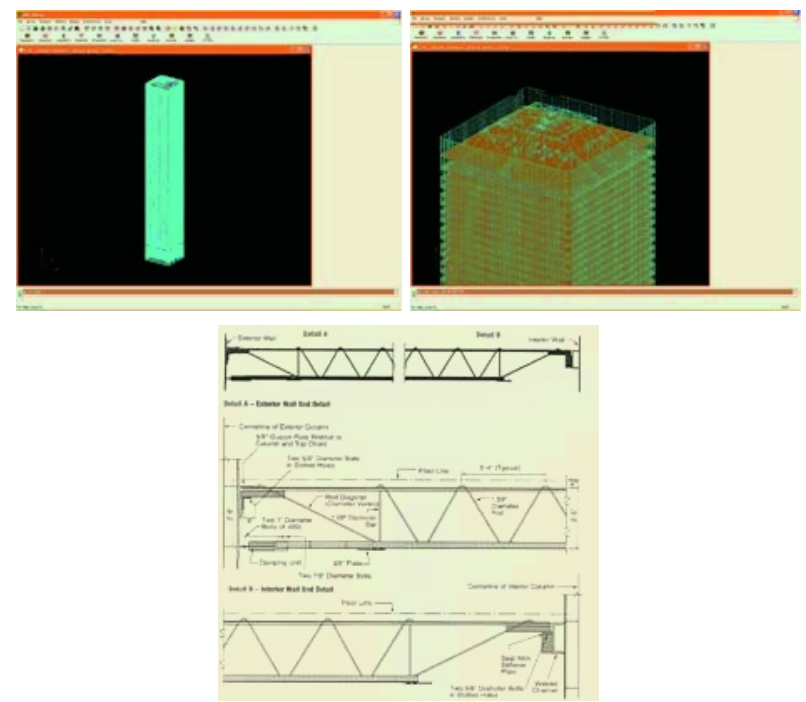

Fig. 5. A high-resolution mesh of the World Trade Center (WTC), developed using detailed blueprints (of the kind shown in bottom panel), which will be used for highfidelity coupled fire-structure simulation of the WTC collapse

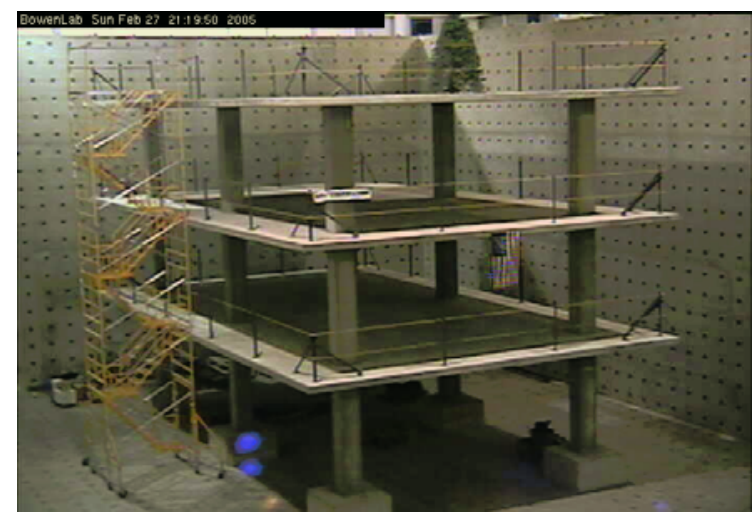

Fig. 6. Image of the test structure $(30 \times 50 \times 35$ feet $)$ at the Bowen Lab for Structural Engineering at Purdue University. The structure will be subject to a variety of external loads and responses will be used to validate model reduction and simulation frameworks. A live web-cam image of this structure is posted at http://newton.ecn.purdue.edu/ ce/Bowen/Webcam/

from $11.5 "$ to $27.5 "$ with a resolution of 0.0048 inches at a frequency of $1250 \mathrm{~Hz}$. These sensors provide RS232 outputs, which we network using RS232/BlueTooth interfaces. Accelerations are measured using the Crossbow Mica2 motes. These devices are networked to an XScale device, which is also equipped with suitable BlueTooth and $802.11 \mathrm{~b}$ interfaces. The entire network is organized hierarchically 
into a scalable, robust, and efficient network. Initial testing of this setup has been completed and the network is now being deployed on the test structure. In the process of this testing and deployment, we have also addressed a number of problems in sensor networking, including multiclass routing, resource-based routing, suitable operating system abstractions and programming interfaces, and power-aware resource management.

\section{Acknowledgements}

The authors would like to acknowledge Prof. Voicu Popescu and Dr. Sami Kilic for their help in generating some of the images in this paper. This work is supported by NSF contract ITR ACI-0324944.

\section{References}

1. B. Carbunar, A. Grama, and J. Vitek. Distributed and dynamic voronoi overlays for coverage detection and distributed hash tables in ad-hoc networks. ICPADS, 2004 .

2. B. Carbunar, A. Grama, J. Vitek, and O. Carbunar. Coverage preserving redundancy elimination in sensor networks. In Proceedings of the 1st IEEE International Conference on Sensor and Ad Hoc Communications and Networks (SECON), Santa Clara, October 2004.

3. Y. Chahlaoui and P. Van Dooren. Benchmark examples for model reduction of linear time invariant dynamical systems. In P. Benner et al., editor, Model Reduction of Dynamical Systems. Springer Verlag, 2004.

4. Y. Chahlaoui and P. Van Dooren. Model reduction of time-varying systems. In P. Benner et al., editor, Model Reduction of Dynamical Systems. Springer Verlag, 2004.

5. Y. Chahlaoui, D. Lemonnier, A. Vandendorpe, and P. Van Dooren. Second-order balanced truncation. Lin. Alg. Appl., 2005. to appear.

6. K. Gallivan, A. Vandendorpe, and P. Van Dooren. Model reduction of mimo systems via tangential interpolation. SIAM J. Matrix Anal. Appl., 26(2):328-349, 2004.

7. C. Hoffmann, S. Kilic, V. Popescu, and M. Sozen. Integrating modeling, visualization and simulation. IEEE Computating in Science and Engineering, pages 52-60, January/February 2004.

8. C. Hoffmann and V. Popescu. Fidelity in visualizing large-scale simulations. Computer-Aided Design, 2005. to appear.

9. S.Kilic, F.Saied, and A.Sameh. Efficient iterative solvers for structural dynamics problems. Computers \& Structures, 82(28):2363-2375, 2004. 